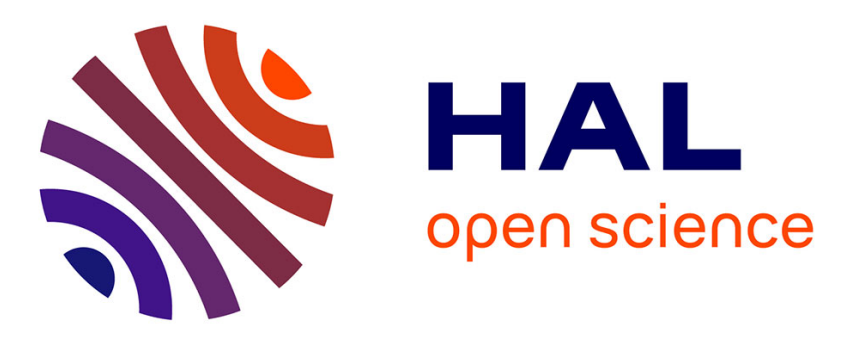

\title{
Road Geometry and Steering Reconstruction for Powered Two Wheeled Vehicles
}

\author{
Majda Fouka, Lamri Nehaoua, Dalil Ichalal, Hichem Arioui, Saïd Mammar
}

\section{To cite this version:}

Majda Fouka, Lamri Nehaoua, Dalil Ichalal, Hichem Arioui, Saïd Mammar. Road Geometry and Steering Reconstruction for Powered Two Wheeled Vehicles. 21st International Conference on Intelligent Transportation Systems (ITSC 2018), Nov 2018, Maui, HI, United States. pp.2024-2029, 10.1109/ITSC.2018.8569422 . hal-02006933

\section{HAL Id: hal-02006933 https://hal.science/hal-02006933}

Submitted on 19 Dec 2019

HAL is a multi-disciplinary open access archive for the deposit and dissemination of scientific research documents, whether they are published or not. The documents may come from teaching and research institutions in France or abroad, or from public or private research centers.
L'archive ouverte pluridisciplinaire HAL, est destinée au dépôt et à la diffusion de documents scientifiques de niveau recherche, publiés ou non, émanant des établissements d'enseignement et de recherche français ou étrangers, des laboratoires publics ou privés. 


\title{
Road Geometry and Steering Reconstruction for Powered Two Wheeled Vehicles
}

\author{
M. Fouka, L. Nehaoua, D. Ichalal, H. Arioui and S. Mammar
}

\begin{abstract}
This paper deals with the estimation of both motorcycle lateral dynamics and road geometry reconstruction. A linear parameter varying (LPV) unknown input observer is designed to estimate the whole motorcycle dynamic states including road banking angles and the rider's steering torque taken into account the variation of the forward velocity. The road bank angle and the lateral slip angle are relevant parameters for improving rider's safety and handling, thus, it is interesting to estimate the road geometry. The observer convergence study is based on Lyapunov theory and the established convergence conditions are expressed in linear matrix inequalities (LMIs) formalism. The main idea consists in getting a set of conditions to design an observer transformed into a polytopic form, which estimates a part of the motorcycle dynamics states independently of some inputs (rider torque) and/or other states (zeros dynamics: roll angle) taken into account the variation of the longitudinal velocity.

Simulation results of experimental test are provided to confirm the efficiency of the proposed design method.
\end{abstract}

Index Terms-Motorcycle dynamics, UI Observer, Lyapunov theory, LMI, Motorcycle safety.

\section{INTRODUCTION}

Major two wheelers vehicle manufacturers, are accelerating Powered Two Wheeled vehicles (PTWv) and advanced rider assistance systems (ARAS) development efforts to get ahead in the race. For this reason, interest in safety problems of PTW vehicles has been growing over the past few years [1] [2]. ARAS systems are very important to improve riders safety and handling in both normal and critical situations. In this context, it is necessary to acquire knowledge on the behavior of the PTWv from the pertinent parameters governing motorcycle dynamics (roll angle, yaw rate, tire cornering forces), involved by riders action (steering torque, steering angle) and/or the infrastructure geometry (road bank angle which is very useful to detect the rollover and sideslip angles to detect a skidding motorcycle). While some states are readily measured with inexpensive sensors (yaw rate), others states must be estimated by more sophisticated means (tire cornering forces). Safety systems nowadays available collect some states from integration of inertial sensors, but this estimation method is combined with errors accumulation and uncertainties from road geometry (slope and bank angles).

Model-based estimators are therefore interesting to overcome previous shortcomings in order to provide estimates of unmeasured states and relevant parameters. In this context,

This work was supported by ANR project : VIROLO++ . All the authors are with the Informatique, Biologie Intgrative et Systmes Complexes Laboratory (IBISC), Evry Val d'Essonne University (UEVE), 40, rue de Pelvoux, 91020 Evry Courcouronne Cedex majda. fouka@univ-evry. fr it seems necessary to estimate the road geometry in order to evaluate the infrastructure impact. One challenge to estimate the road bank angle is to improve estimation of the side slip angle on tilted road surfaces, the road banking have a direct influence on motorcycle lateral motions (lateral acceleration measurement). Thus, knowing the road bank angle allows us to achieve safety speeds and desired control on difficult road conditions, because part of the centrifugal force is compensated by this inclination. Several methods were proposed to estimate and identify the motorcycle dynamics states and parameters such as, ([3]-[13]). Concerning lateral estimation most of cited papers consider restrictive assumptions, regarding riding motorcycle practices and/or a constant longitudinal speed, the road geometry and tire-road contact has often been neglected. To the best of our knowledge, the simultaneous estimation of the lateral dynamics and the road geometry was treated on vehicles and those methods developed for fourwheeled vehicles are not necessarily adequate for motorcycle [14].

The present paper proposes an unknown input observer (UIO) considering linear parameter-varying (LPV) motorcycle model and a certain number of valid measurements, taking into account the variations in the longitudinal speed, the model is transformed into polytopic form by following the sector non-linearity approach [15] coupled with model inversion. Using Lyapunov concept and LMI region for performance enhancement, the observer is able to estimate, asymptotically, lateral motorcycle dynamics and to reconstruct unknown inputs (roll angle and steering torque) with bank angle reconstruction. The tire's dynamic behavior is taken into account when evaluating the generated lateral tire force. In order to show the effectiveness of the estimation method, a validation test were carried out on an instrumented motorcycle in realistic riding situations.

The paper is organized as follows. Sec. II, describes and discusses the motorcycle out of plane model. Sec. III, illustrates the observer design and presents the convergence analysis. Sec. IV, discuss the results, compared to real experimental data and evaluate the estimated model. Finally, sec. V, concludes the paper.

\section{The Out-Of-Plane Motorcycle Dynamics}

In this work, the Out-Of-Plane motorcycle motions are modeled as presented in Sharp's 71 model [16] [17], due essentially to the effect of lateral forces, including tire relaxation for the stability of the Sharp's model, lateral displacement, the yaw and roll motions of the main frame under rider's steering actions. We consider the four-dimensional 
model (4 DoF) with road bank angle consideration and nonlinear tire characteristics. Figure (1) shows the motorcycle and the road geometry. This paper extends the earlier results

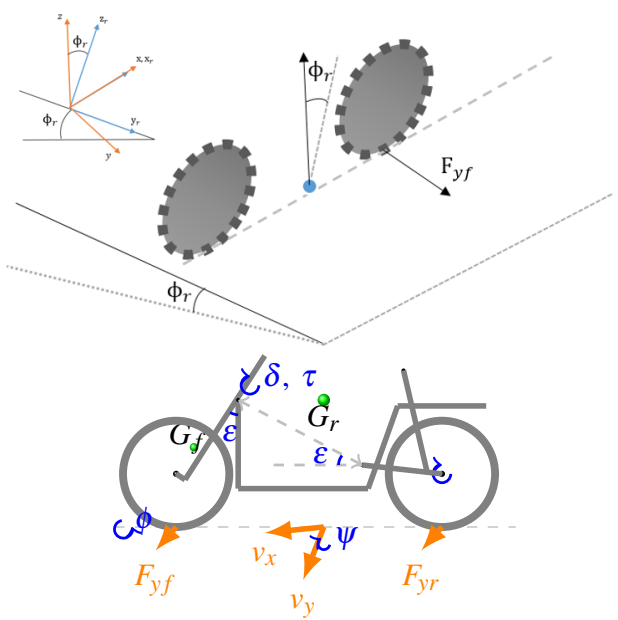

Fig. 1: Motorcycle geometry with road banking angle.

in Sharp's 71 model, taking into account the road bank angle, denoted $\phi_{r}$, it has the effect of keeping the motorcycle in its path, to avoid the overturning of motorcycle, this force is noted $F_{\phi_{r}}=M g \sin \left(\phi_{r}\right)$.

Assumption 1: Considering the following assumptions:

- Assume that the road bank angle is constant piecewise or varies slowly.

- The rider is rigidly attached to the main frame.

Under these assumptions, the motions of the motorcycle can be described by the following equations

$$
\left\{\begin{array}{l}
e_{33} \dot{v}_{y}+e_{34} \ddot{\psi}+e_{35} \ddot{\phi}+e_{36} \ddot{\delta}=m_{34} \dot{\psi}+F_{y f}+F_{y r}-M g \sin \left(\phi_{r}\right) \\
e_{34} \dot{v}_{y}+e_{44} \ddot{\psi}+e_{45} \ddot{\phi}+e_{46} \ddot{\delta}=m_{44} \dot{\psi}+m_{45} \dot{\phi}+m_{46} \dot{\delta}+ \\
m_{47} F_{y f}+m_{48} F_{y r} \\
e_{35} \dot{v}_{y}+e_{45} \ddot{\psi}+e_{55} \ddot{\phi}+e_{56} \ddot{\delta}=m_{51} \sin \left(\phi+\phi_{r}\right)+m_{52} \sin (\delta)+ \\
m_{54} \dot{\psi}+m_{56} \dot{\delta} \\
e_{36} \dot{v}_{y}+e_{46} \ddot{\psi}+e_{56} \ddot{\phi}+e_{66} \ddot{\delta}=m_{61} \sin \left(\phi+\phi_{r}\right)+m_{62} \sin (\delta)+ \\
m_{64} \dot{\psi}+m_{65} \dot{\phi}+m_{66} \dot{\delta}+m_{67} F_{y f}+\tau \\
\dot{F}_{y f}=m_{71}\left(\phi+\phi_{r}\right)+m_{72} \delta+m_{73} v_{y}+m_{74} \dot{\psi}+m_{76} \dot{\delta}+m_{77} F_{y f} \\
\dot{F}_{y r}=m_{81}\left(\phi+\phi_{r}\right)+m_{83} v_{y}+m_{84} \dot{\psi}+m_{88} F_{y r}
\end{array}\right.
$$

Where $(\phi, \delta, \psi, \dot{\phi}, \dot{\delta}, \dot{\psi})$ denote the roll, steering, yaw angles and respectively their time derivatives, whereas $\left(\phi_{r}\right.$, $v_{y}$ ) are respectively the road bank angle and lateral velocity, $F_{y f}$ and $F_{y r}$ are the cornering front and rear forces respectively, and $\tau$ is the torque applied to the handle bar. For further details on the motorcycle parameters $\left(e_{i j}, m_{i j}\left(v_{x}\right)\right)$ and expressions refer to tables I and II.

Note that the roll, road banking angles, lateral speed and the tire forces in the dynamic state are hard to measure. For the PTWv, we measure ( $\left.\dot{\phi}, \dot{\psi}, v_{x}, \delta, a_{y}\right)$ and its timederivative rate.
Remark 1: To be more consistent with realistic constraints, we consider the road banking, roll angle and the steering torque as unknown inputs.

\section{A. Motorcycle LPV Model}

Two wheelers vehicle is stable only for a range of forward velocities, contrarily to a standard vehicle with four wheels. Assume that the premise variables $\rho=v_{x}$ is the longitudinal speed, real time accessible from GPS, its first time derivative exists and is noted $\dot{\rho}(t)$ can be obtained from hight differentiator or from forward acceleration and it will be used in the observer design.

Consider, the parameter vector $(\rho, \dot{\rho}) \in \Delta$, where $\Delta$ define hyper-rectangles defined by:

$\Delta=\left\{(\rho, \dot{\rho}) \in R \mid \rho_{\text {min }} \leq \rho(t) \leq \rho_{\text {max }}, \bar{\rho}_{\text {min }} \leq \dot{\rho}(t) \leq \bar{\rho}_{\text {max }}\right\}$

The motorcycle dynamic model can be expressed by the following Linear Parameter Varying (LPV) structure :

$$
\left\{\begin{array}{c}
\dot{x}(t)=A(\rho) x(t)+D_{\phi}(\rho) \phi(t)+D_{\phi_{r}}(\rho) \phi_{r}(t)+B \tau(t) \\
y=C x(t)
\end{array}\right.
$$

where $x=\left[\delta, v_{y}, \dot{\psi}, \dot{\phi}, \dot{\delta}, F_{y f}, F_{y r}\right]^{T} \in \mathbb{R}^{n}$ denotes the state vector, $v(t)=\left[\begin{array}{lll}\phi & \phi_{r} & \tau\end{array}\right]^{T} \in \mathbb{R}^{p}$ is the unknown part of the model, $y \in \mathbb{R}^{n_{y}}$ is the vector of measures. The matrices $A(\rho) \in \mathbb{R}^{n \times n}, D_{\phi}(\rho) \in \mathbb{R}^{n \times 1}, D_{\phi_{r}}(\rho) \in \mathbb{R}^{n \times 1}$, are parameter varying, while the matrices $C \in \mathbb{R}^{n_{y} \times n}$ and $B \in \mathbb{R}^{n \times m}$ are constants. The numerical values of the matrices are given in the appendix.

Let, $G(\rho)=\left[\begin{array}{lll}D_{\phi}(\rho) & D_{\phi_{r}}(\rho) & B\end{array}\right], D_{\phi}(\rho)$ is the vector corresponding to the roll state and $D_{\phi_{r}}(\rho)$ is the vector corresponding to the road banking angle.

The model (3) is rewritten as follow:

$$
\left\{\begin{array}{c}
\dot{x}(t)=A(\rho) x(t)+G(\rho) v(t) \\
y=C x(t)
\end{array}\right.
$$

One can remark that $\operatorname{dim}(y(t))>\operatorname{dim}(v(t))$ is satisfied.

\section{OBSERVER DESIGN}

In this section we explain how to design an unknown input observer (UIO) considering LPV system, to state estimation. The approach considers Lyapunov theory associated with LMI tools to guarantee the asymptotically convergence to zero of the state estimation errors.

Assumption 2: To design a stable unknown input observer, the well-known conditions for the existence of the UIO are given by the following theorem [18]:

1) The pair $(A(\rho), C)$ is detectable $\forall \rho(t) \in \Delta$.

2) Matching condition: $\operatorname{rank}(C G(\rho))=$ $\operatorname{rank}(G(\rho)), \quad \forall \rho(t) \in \Delta$, is satisfied.

Let us denote $\hat{x}(t)$ and $\hat{y}(t)$ the estimated state and output vectors respectively. Consider the following unknown input observer [20]:

$$
\left\{\begin{array}{lcc}
\dot{z}(t) & = & N(\rho, \dot{\rho}) z(t)+L(\rho, \dot{\rho}) y(t) \\
\hat{x}(t) & = & z(t)-H(\rho) y(t)
\end{array}\right.
$$


The matrices $N(\rho, \dot{\rho}) \in \mathbb{R}^{n \times n}, L(\rho, \dot{\rho}) \in \mathbb{R}^{n \times n_{y}}$ and $H(\rho) \in$ $\mathbb{R}^{n \times n_{y}}$ of the observer are to be determined to ensure asymptotic state estimation error even in the presence of UI.

According to equations $(4,5)$, the state estimation error is given by:

$$
e(t)=x(t)-\hat{x}(t)=\underbrace{(I+H(\rho) C)}_{P(\rho(t))} x(t)-z(t)
$$

The state estimation error obeys to the following differential equation:

$$
\begin{aligned}
\dot{e}(t) & =\dot{P}(\rho, \dot{\rho}) x+P(\rho) \dot{x}-\dot{z} \\
& =N(\rho, \dot{\rho}) e+(\dot{P}(\rho, \dot{\rho})+P(\rho) A(\rho)-N(\rho, \dot{\rho}) P(\rho) \\
& -L(\rho, \dot{\rho}) C) x(t)+P(\rho) G(\rho) v(t)
\end{aligned}
$$

With $z(t)=P(\rho) x(t)-e(t)$.

If the following conditions hold:

$$
\begin{aligned}
\dot{P}(\rho, \dot{\rho})+P(\rho) A(\rho)-N(\rho, \dot{\rho}) P(\rho)-L(\rho, \dot{\rho}) C & =0 \\
P(\rho) G(\rho) & =0
\end{aligned}
$$

The estimation error dynamics will be reduced to:

$$
\dot{e}(t)=N(\rho, \dot{\rho}) e(t)
$$

In which, $N(\rho, \dot{\rho})$ must be Hurwitz.

\section{A. Convergence study}

In this paper, the stability analysis of the observer (5) is studied in order to obtain the observer gains that satisfy the LMI conditions. This analysis is performed by using a quadratic Lyapunov function as follows :

$$
V(e(t))=e(t)^{T} Q e(t), \quad Q=Q^{T}>0
$$

By using the error dynamics in equation (10), the time derivative of the Lyapunov function can be written by the following expression:

$$
\dot{V}(t)=e(t)^{T}\left(N(\rho, \dot{\rho})^{T} Q+Q N(\rho, \dot{\rho})\right) e(t)
$$

Equations (8) to (9) form a set of an LMI conditions to be resolved to compute the observer gains. One replace the matrix $P(\rho)=I+H(\rho) C$ in the equality (8) which leads to:

$$
\begin{array}{r}
\dot{P}(\rho, \dot{\rho})+P(\rho) A(\rho)-N(\rho, \dot{\rho}) P(\rho)-L(\rho, \dot{\rho}) C=0 \\
\underbrace{\dot{P}(\rho, \dot{\rho})+P(\rho) A(\rho)}_{\Gamma(\rho, \dot{\rho})}-N(\rho, \dot{\rho})-(\underbrace{N(\rho, \dot{\rho}) H(\rho)+L(\rho, \dot{\rho})}_{K(\rho, \dot{\rho})}) C=0
\end{array}
$$

Thus,

$$
N(\rho, \dot{\rho})=\Gamma(\rho, \dot{\rho})-K(\rho, \dot{\rho}) C
$$

$\Gamma(\rho, \dot{\rho})$ and $K(\rho, \dot{\rho})$ are function of the parameters varying signals $(\rho(t), \dot{\rho}(t))$.

The dynamics of the state estimation error's become:

$$
\dot{e}(t)=(\Gamma(\rho, \dot{\rho})-K(\rho, \dot{\rho}) C) e(t)
$$

The time derivative of the Lyapunov function is written by the following expression:

$$
\begin{aligned}
\dot{V} & =e^{T}\left((\Gamma(\rho, \dot{\rho})-K(\rho, \dot{\rho}) C)^{T} Q+Q(\Gamma(\rho, \dot{\rho})-K(\rho, \dot{\rho}) C)\right) e \\
& =e^{T}\left(\Gamma(\rho, \dot{\rho})^{T} Q-C^{T} R(\rho, \dot{\rho})^{T}+Q \Gamma(\rho, \dot{\rho})-R(\rho, \dot{\rho}) C\right) e
\end{aligned}
$$

where $R(\rho, \dot{\rho})=Q K(\rho, \dot{\rho})$

Theorem 1: The state estimation error converges asymptotically toward zero if there exist a symmetric positive definite matrix $Q \in \mathbb{R}^{n \times n}$ and a matrix $R \in \mathbb{R}^{n \times n y}$ satisfying the Lyapunov inequality:

$$
\Gamma(\rho, \dot{\rho})^{T} Q+Q \Gamma(\rho, \dot{\rho})-R(\rho, \dot{\rho}) C-C^{T} R(\rho, \dot{\rho})^{T}<0
$$

It follows that $V(e)=e^{T} Q e>0$ defines a common quadratic Lyapunov function for the observer.

Note that the Lyapunov inequality depend on the varying parameters, in order to derive LMI conditions that ensure the asymptotic convergence of the state estimation error, the polytopic approach is used to transform the inequality 14 .

\section{B. Polytopic Representation}

The nonlinearities in the observer are taken into account by considering a polytopic representation to define a set of Linear Time Invariant (LTI) models interconnected with nonlinear weighting functions $\eta_{i}($.$) which are known and$ they must satisfy the following convex sum property [15]:

$$
\left\{\begin{array}{l}
\sum_{i=1}^{r} \eta_{i}(\rho(t), \dot{\rho}(t))=1 \\
0 \leq \eta_{i}(\rho(t), \dot{\rho}(t)) \leq 1
\end{array} \forall i=\{1,2, . ., r\}\right.
$$

$r$ represents the number of local submodels defines by $r=$ $2^{n_{\rho}}$ where $n_{\rho}=2$ is the number of non-linearities (for more details, please refer to [15], [20], [21]). Then the sector nonlinear approach is used to transform the problem into a (TS) form :

$$
\left\{\begin{array}{c}
\Gamma(\rho, \dot{\rho})=\dot{P}(\rho, \dot{\rho})+P(\rho) A(\rho)=\sum_{i=1}^{r} \eta_{i}(\rho, \dot{\rho}) \Gamma_{i} \\
K(\rho, \dot{\rho})=\sum_{i=1}^{r} \eta_{i}(\rho, \dot{\rho}) K_{i},(\rho, \dot{\rho}) \in \Delta
\end{array}\right.
$$

\section{Eigenvalue Assignment Problem}

The performances of the observer can be improved by pole assignment in an LMI region to ensure an acceptable transient response, given by the stability margin $\alpha$ in a subset $\Theta$ of the complex plane such that the matrix $\Gamma_{i}$ is said $\Theta_{i^{-}}$ Stable when its spectrum $\lambda\left(\Gamma_{i}\right)$ belongs to region $\Theta_{i}$ [19].

$$
\Theta_{i}=\left\{z=\left(x_{z}+i \cdot y_{z} \in \mathbb{C} \mid \operatorname{Re}(z) \leq-\alpha \Leftrightarrow z+\bar{z}+2 \alpha<0\right\}\right.
$$

where $\mathbb{C}$ is the set of complex numbers, and $\bar{z}$ denotes the complex conjugate of $z$. Using the convex sum property of the weighting functions and from (14), sufficient LMI conditions ensuring asymptotic stability in LMI region are obtained as follows :

$$
\Gamma_{i}^{T} Q+Q \Gamma_{i}-C^{T} R^{T}-R C+2 \alpha Q<0, i \in(1, \ldots, r)
$$

Where, $R_{i}=Q K_{i}$ and $Q=Q^{T}>0$. This equation provides a way of ensuring the eigenvalues of a matrix within a specific region.

1) The LMI problem includes the following condition to compute the matrices $H(\rho)$ as follows:

$$
\begin{gathered}
\left\{\begin{array}{c}
P(\rho) G(\rho)=0 \\
P(\rho)=I+H(\rho) C
\end{array}\right. \\
H(\rho)=-G(\rho)(C G(\rho))^{\dagger}
\end{gathered}
$$

The solution of this equation depends on the rank of matrix $C G(\rho), H(\rho)$ exists if $\operatorname{rank}(C G(\rho))=$ 
$\operatorname{rank}(G(\rho)) \forall \rho$. Since $C G(\rho)$ is of full column rank, $(C G(\rho))^{\dagger}=\left[(C G(\rho))^{T}(C G(\rho))\right]^{-1}(C G(\rho))^{T}$ is the left pseudo-inverse of the matrix $(C G(\rho))$.

2) The gains of the observer $N(\rho, \dot{\rho})$ and $L(\rho, \dot{\rho})$ are computed as follows:

$$
\begin{gathered}
K(\rho, \dot{\rho})=Q^{-1} R(\rho, \dot{\rho}) \\
N(\rho, \dot{\rho})=\Gamma(\rho, \dot{\rho})-K(\rho, \dot{\rho}) C \\
L(\rho, \dot{\rho})=K(\rho, \dot{\rho})-N(\rho, \dot{\rho}) H(\rho)
\end{gathered}
$$

for more details, please refer to [21].

\section{Unknown Input estimation}

After estimating the states of the system, the unknown inputs can be estimated by a simple dynamic system inversion. From the output equation $y=C x$, one can write:

$$
\dot{y}=C A(\rho) x(t)+C G(\rho) v(t)
$$

Since the condition $\operatorname{rank}(C G(\rho))=\operatorname{rank}(G(\rho))$, is satisfied, it follows:

$$
v(t)=\left[\begin{array}{c}
\phi(t) \\
\phi_{r}(t) \\
\tau(t)
\end{array}\right]=(C G(\rho))^{\dagger}(\dot{y}(t)-C A(\rho) x)
$$

When the state estimation error $e(t)$ converges to zero, we have $\hat{x}(t) \rightarrow x(t)$, then the following UI estimation $\hat{v}$ is obtained by the following equation :

$$
\hat{v}(t)=(C G)^{\dagger}(\dot{y}(t)-C A(\rho) \hat{x}(t))
$$

In which, the convergence of $\hat{v}$ toward $v$ can be analyzed by defining the unknown input estimation error

$$
e_{v}(t)=v(t)-\hat{v}(t)=-(C G)^{\dagger} C A(\rho) e(t), \quad \forall \rho(t) \in \Delta
$$

knowing that $e(t)$ converges asymptotically to zero, then $e_{v}(t)$ also converges asymptotically to zero.

According to Lyapunov formulation, the state and unknown input errors converge asymptotically to zero in order to achieve an accurate estimation of the states of the motorcycle and the torque applied on the handlebar.

\section{EXPERIMENTAL RESUlts}

This section aims to present experimental results using a scenario realized on urban scenic road and normal riding behavior to validate the proposed observer. The instrumented motorcycle is an Electric Scooter Peugeot model (figure 2) (please refer to [22] for data set and experimentation).
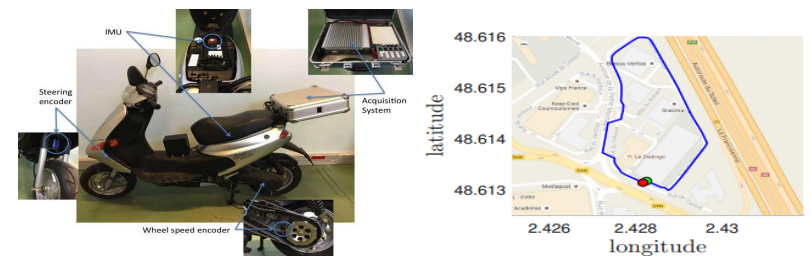

Fig. 2: Instrumented Scooter of IBISC Lab and Vehicle trajectory

The simulations are conducted to illustrate the efficiency of the presented approach, the optimization problem under
LMI conditions is solved using Yalmip toolbox under Matlab software. The observer estimate the lateral dynamics using the measured states $\left(\dot{\phi}, \dot{\psi}, a_{y}\right)$ given by the inertial unit and $\delta$ obtained from the steering encoder, longitudinal speed obtained from GPS data. The scenario is considered with a varying longitudinal velocity from $7 \mathrm{~m} / \mathrm{s}$ on $18 \mathrm{~m} / \mathrm{s}$, depicted in (fig. 3 ).

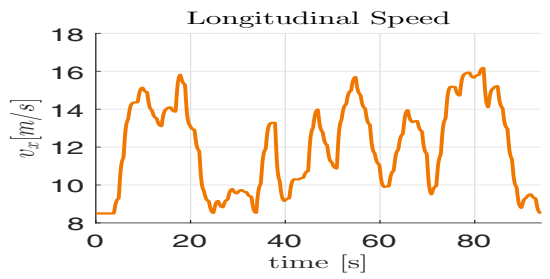

Fig. 3: Longitudinal velocity

The actual signals given by the encoder and the IMU required in the observer design are depicted in (fig. 4 ).
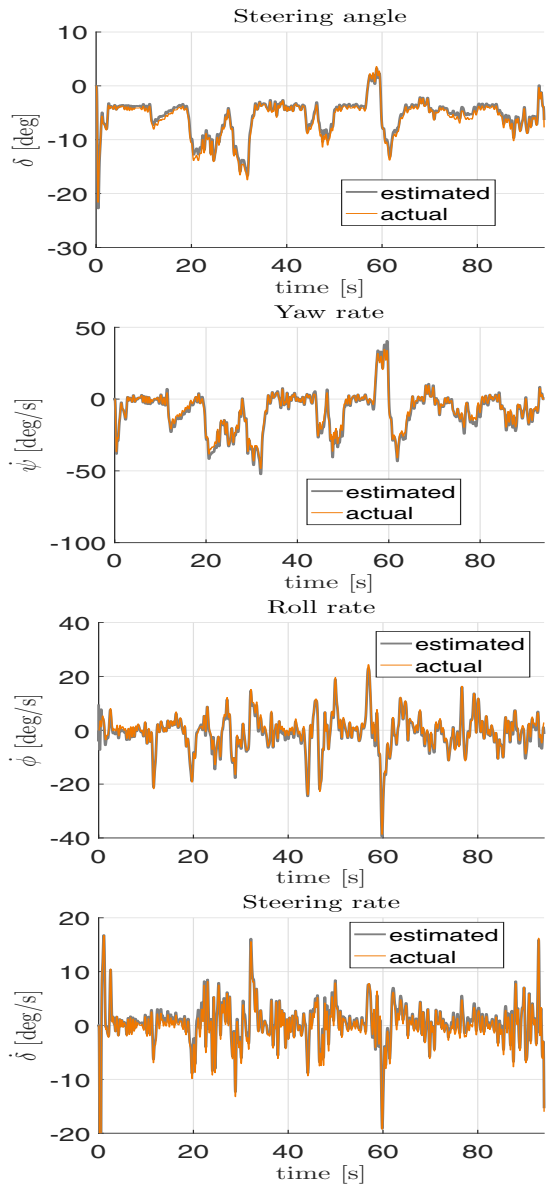

Fig. 4: States estimation (gray) compared to actual measurement (orange).

Figures (5) shows the states estimation of the unmeasurable states from the lateral dynamic model, whereas (fig. 
6) illustrate the estimation unknown inputs from the model inversion.
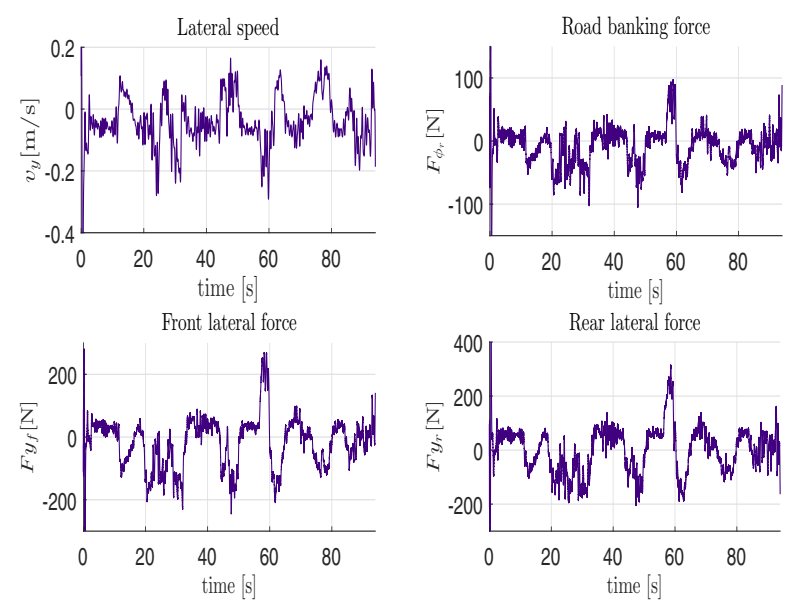

Fig. 5: Unmeasurable states estimation of scooter.
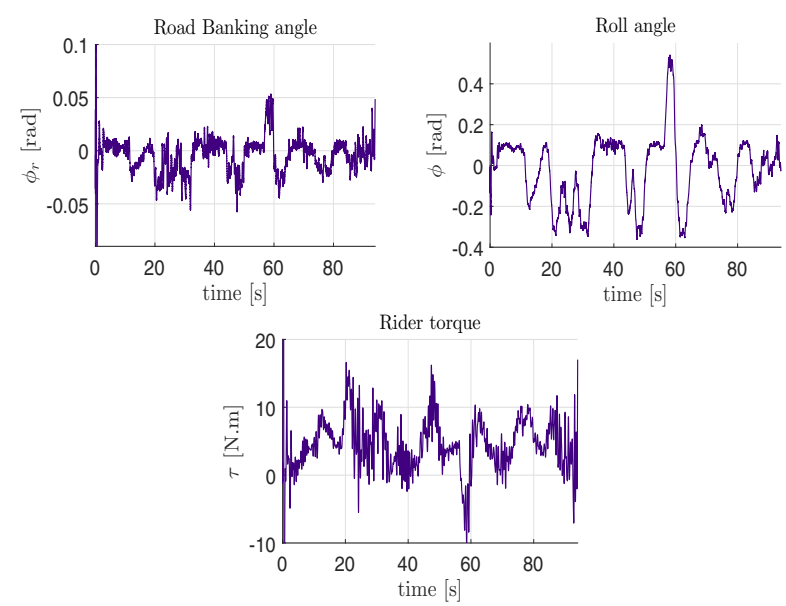

Fig. 6: States estimation of scooter from model inversion.

\section{A. Validated dynamic states}

Since the actual state $v_{y}$ and $F_{y f}, F_{y r}, F_{\phi_{r}}$ are unknown, the state estimation cannot be checked directly as it was done in figure 4 in the above simulation. To validate the estimation, one can use the estimated state $v_{y}$ and $\left(F_{y f}, F_{y r}, F_{\phi_{r}}\right)$ to construct the lateral acceleration from equations (22-23) to compare with the measured acceleration (given by the IMU) in figures 7 .

$$
\begin{aligned}
M \hat{a}_{y} & =\hat{F}_{y f}+\hat{F}_{y r}-\hat{F}_{\phi_{r}} \\
\hat{a}_{y} & =\hat{v_{y}}+v_{x} \dot{\psi} \\
\phi_{I M U} & =\phi+\phi_{r}
\end{aligned}
$$

Figure 7 show the actual lateral acceleration compared to the sum of the estimated lateral forces and also to the estimated with lateral motion, the roll measure given by the IMU can also be used to verified the sum of $\left(\phi\right.$ and $\left.\phi_{r}\right)$ in equation (24) :

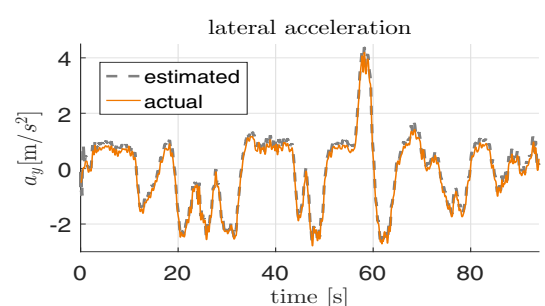

(a) Validate $F_{y f}, F_{y r}, F_{\phi_{r}}$ estimated (gray) from the lateral acceleration (orange)

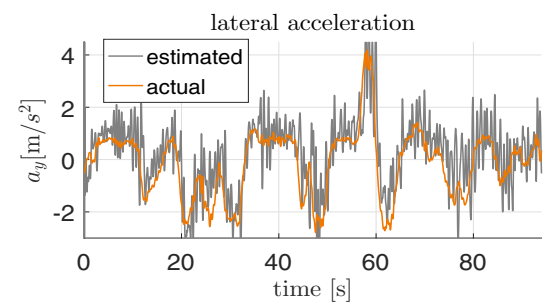

(b) Validate $v_{y}$ estimated (gray) from the lateral acceleration (orange)

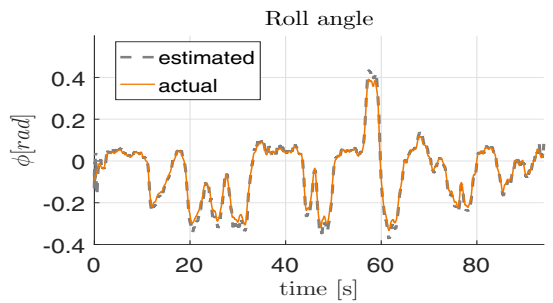

(c) Validate $\phi, \phi_{r}$ estimated (gray) from the Roll angle measurement (orange)

Fig. 7: State estimation validation $: v_{y}, F_{y f}, F_{y r}, F_{\phi_{r}}$ from the lateral acceleration and the additional measurement; and $\phi, \phi_{r}$ from IMU roll angle.

According to the above simulation results, it can be seen that the observer has a rapid transient phase and an acceptable convergence to the estimated value, the estimated model gives a good representation of the actual states. We see that all the state and variables are well estimated except the lateral velocity which has significant error estimation, but it is still acceptable. The lateral velocity is difficult to estimate accurately. However, because of its low value compared to longitudinal velocity this error does not affect the performances of the roll angle estimation. The roll and steer angles $\left(\phi+\phi_{r}, \delta\right)$ and also the lateral forces are well estimated, there are some differences at the peak due to modeling uncertainties, between the scooter and the estimated model. The model used for the observer design does not take into account large roll angles but the observer still gives acceptable results. Finally, figures shows the ability of the designed observer to well recover simultaneously the motorcycle dynamics, unknown inputs and the road banking angle.

\section{CONClusion}

In this paper, an estimation of out of plane motorcycle model, road feature and unknown inputs (rider's torque and 
roll angle) have been proposed using an unknown input observer associated with model inversion. The design method takes into account the forward speed as a linear parameter varying. Sufficient conditions for the existence of the estimator are given in terms of linear matrix inequalities (LMIs). The performance of the resulting observer has been evaluated by experimental validation using a riding scenario. Simulation results are provided which illustrate the effectiveness of the proposed observer in estimating the states, unknown input and road geometry, the observer result demonstrate that the observer gives reliable estimations.

\section{APPENDIX}

\section{A. System's parameters description and numerical values}

TABLE I: motorcycle parameters

\begin{tabular}{ll}
\hline Parameters & \\
\hline$M_{f}, M_{r}, M$ & mass of the front frame, the rear frame \\
$K$ & and the whole motorcycle \\
$Z_{f}, Z_{r}$ & damper coefficient of the steering \\
$C_{f 1}, C_{r 1}$ & front and rear vertical forces \\
$C_{f 2}, C_{r 2}$ & front and rear tire cornering stiffness \\
$\sigma_{f}, \sigma_{r}$ & coefficients of relaxation of the front \\
& and rear pneumatic forces \\
$j, h, k, e, l_{f}, l_{r}$ & linear dimensions \\
$i_{f y}, i_{r y}$ & polar moment of inertia of front and rear wheels \\
$R_{f}, R_{r}$ & radius of front and rear wheels \\
$\varepsilon$ & caster angle \\
$K$ & damper coefficient of the steering mechanism \\
$\eta$ & mechanical trail \\
$I_{f x}, I_{r x}, I_{f z}, I_{r z}$ & front and rear frame inertias about $X / Z$ axis \\
$C_{r x z}$ & rear frame product of inertia, $X$ and $Z$ axis \\
\hline
\end{tabular}

TABLE II: Motorcycle parameters expressions

$e_{33}=M, e_{35}=M_{f} j+M_{r} h, e_{46}=M_{f} e k+I_{f z} \cos \varepsilon, e_{34}=M_{f} k$,
$e_{44}=M_{f} k^{2}+I_{r z}+I_{f x} \sin ^{2} \varepsilon+I_{f z} \cos ^{2} \varepsilon, m_{44}=-M_{f} k v_{x}, e_{36}=M_{f} e$
$e_{45}=M_{f} j k-C_{r x z}+\left(I_{f z}-I_{f x}\right) \sin \varepsilon \cos \varepsilon, m_{34}=-M v_{x} m_{45}=\frac{i_{f y}}{R_{f}}+\frac{i_{r y}}{R_{r}}$,
$m_{46}=\frac{i_{f y}}{R_{f}} \sin \varepsilon v_{x} e_{55}=M_{f} j^{2}+M_{r} h^{2}+I_{r x}+I_{f x} \cos ^{2} \varepsilon+I_{f z} \sin ^{2} \varepsilon$,
$e_{56}=M_{f} e j+I_{f z} \sin \varepsilon, m_{47}=l_{f}, m_{48}=-l_{r}, m_{51}=\left(M_{f} j+M_{r} h\right) g$,
$m_{54}=-\left(M_{f} j+M_{r} h+\frac{i_{f y}}{R_{f}}+\frac{i_{r y}}{R_{r}}\right) v_{x}, m_{56}=-\frac{i_{f y}}{R_{f}} \cos \varepsilon v_{x}$,
$m_{52}=M_{f} e g-\eta Z_{f}, m_{61}=M_{f} e g-\eta * Z_{f}, m_{62}=\left(M_{f} e g-e t a Z_{f}\right) \sin (\varepsilon)$
$e_{66}=I_{f z}+M_{f} e^{2}, m_{64}=-\left(M_{f} e+\frac{i_{f y}}{R_{f}} \sin \varepsilon\right) v_{x}, m_{65}=\frac{i_{f y}}{R_{f}} \cos (\varepsilon) v_{x}$,
$m_{66}=-K, m_{67}=-\eta, m_{71}=\frac{C_{f 2}}{\sigma_{f}} v_{x}, m_{73}=-\frac{C_{f 1}}{\sigma_{f}}, m_{74}=-\frac{C_{f 1}}{\sigma_{f}} l_{f}$,
$m_{72}=\frac{\left(C_{f 1} \cos (\varepsilon)+C_{f 2} \sin (\varepsilon)\right)}{\sigma_{f}} v_{x}, m_{76}=\frac{C_{f 1}}{\sigma_{f}} \eta ; m_{77}=-\frac{1}{\sigma_{f}} v_{x}, m_{81}=\frac{C_{r 2}}{\sigma_{r}} v_{x}$,
$m_{83}=-\frac{C_{r 1}}{\sigma_{r}}, m_{84}=\frac{C_{r 1}}{\sigma_{r}} l_{r}, m_{88}=-\frac{1}{\sigma_{r}} v_{x}$,$$
\begin{gathered}
E=\left[\begin{array}{ccccccc}
1 & 0 & 0 & 0 & 0 & 0 & 0 \\
0 & e_{33} & e_{34} & e_{35} & e_{36} & 0 & 0 \\
0 & e_{34} & e_{44} & e_{45} & e_{46} & 0 & 0 \\
0 & e_{35} & e_{45} & e_{55} & e_{56} & 0 & 0 \\
0 & e_{36} & e_{46} & e_{56} & e_{66} & 0 & 0 \\
0 & 0 & 0 & 0 & 0 & 1 & 0 \\
0 & 0 & 0 & 0 & 0 & 0 & 1
\end{array}\right], \bar{B}=\left[\begin{array}{l}
0 \\
0 \\
0 \\
0 \\
0 \\
1 \\
0 \\
0
\end{array}\right] \bar{D}_{\phi}(\rho)=\left[\begin{array}{c}
0 \\
0 \\
0 \\
m_{51} \\
m_{61} \\
m_{71} \\
m_{81}
\end{array}\right] \\
\bar{D}_{\phi_{r}}(\rho)=\left[\begin{array}{c}
0 \\
m g h \\
0 \\
m_{51} \\
m_{61} \\
m_{71} \\
m_{81}
\end{array}\right] M(\rho)=\left[\begin{array}{ccccccccc}
0 & 0 & 0 & 0 & 1 & 0 & 0 \\
0 & 0 & m_{34} & 0 & 0 & 1 & 1 \\
0 & 0 & m_{44} & m_{45} & m_{46} & m_{47} & m_{48} \\
m_{52} & 0 & m_{54} & 0 & m_{56} & 0 & 0 \\
m_{62} & 0 & m_{64} & m_{65} & m_{66} & m_{67} & 0 \\
m_{72} & m_{73} & m_{74} & 0 & m_{76} & m_{77} & 0 \\
0 & m_{83} & m_{84} & 0 & 0 & 0 & m_{88}
\end{array}\right]
\end{gathered}
$$

Where, the matrix $A(\rho)=E^{-1} M(\rho), D_{\phi}(\rho)=E^{-1} \bar{D}_{\phi}(\rho)$, $D_{\phi_{r}}(\rho)=E^{-1} \bar{D}_{\phi_{r}}(\rho), B=E^{-1} \bar{B}$.

\section{REFERENCES}

[1] B. Evangelos, Saferider project, French National Agency of Reaserch, Tech. Rep., 2010.

[2] N. Haworth, P. Rowden, B. Watson, D. Wishart, L. Buckley, and K. Greig, Motorcycle rider safety project, The Centre for Accident Research and Road Safety Queensland, Tech. Rep., 2012.

[3] Panzani, G., Corno, M., Savaresi, S. M. (2012). Longitudinal velocity estimation in single-track vehicles. IFAC Proceedings Volumes, 45(16), 1701-1706.

[4] Boniolo, I., Savaresi, S. M., Tanelli, M. (2012, May). Lean angle estimation in two-wheeled vehicles with a reduced sensor configuration. In Circuits and Systems (ISCAS), 2012 IEEE International Symposium on (pp. 2573-2576). IEEE.

[5] Corno, M., Panzani, G., Savaresi, S. M. (2013). Traction-controloriented state estimation for motorcycles. IEEE Transactions on Control Systems Technology, 21(6), 2400-2407. ,

[6] Dabladji, M. E. H., Ichalal, D., Arioui, H., Mammar, S. (2016). Unknown-input observer design for motorcycle lateral dynamics: Ts approach. Control Engineering Practice, 54, 12-26.

[7] Fouka, M., Nehaoua, L., Arioui, H., Mammar, S. MUTIPLEGRADIENT DESCENT ALGORITHM FOR PARAMETRIC IDENTIFICATION OF A POWERED TWO-WHEELED VEHICLES.

[8] Fouka, M., Damon, P. M., Nehaoua, L., Arioui, H., Mammar, S. (2017). Parametric Identification of a Powered Two-Wheeled Vehicles: Algebraic Approach

[9] Nehaoua, L., Ichalal, D., Arioui, H., Davila, J., Mammar, S., Fridman, L. M. (2014). An unknown-input HOSM approach to estimate lean and steering motorcycle dynamics. IEEE Transactions on Vehicular Technology, 63(7), 3116-3127.

[10] Ichalal, D., Dabladji, H., Arioui, H., Mammar, S., Nehaoua, L. (2013, June). Observer design for motorcycle lean and steering dynamics estimation: a takagi-sugeno approach. In American Control Conference (ACC), 2013 (pp. 5654-5659). IEEE.

[11] A. P. Teerhuis and S. T. H. Jansen, Motorcycle state estimation for lateral dynamics, Vehicle System Dynamics, vol. 50, no. 8, pp. 12611276, 2012.

[12] P. De Filippi, M. Corno, M. Tanelli, and S. Savaresi, Single-sensor control strategies for semi-active steering damper control in twowheeled vehicles, Vehicular Technology, IEEE Transactions on, vol. 61, no. 2, pp. 813820, 2011.

[13] Slimi, H., Arioui, H., Mammar, S. (2012, July). Dynamique limite pour systmes d'alertes aux conducteurs des deux-roues motoriss. In Septime Confrence Internationale Francophone d'Automatique.

[14] Dahmani, H., Chadli, M., Rabhi, A., El Hajjaji, A. (2011, June). Vehicle dynamics and road geometry estimation using a TakagiSugeno fuzzy observer with unknown inputs. In Intelligent Vehicles Symposium (IV), 2011 IEEE (pp. 272-277). IEEE.

[15] Tanaka, K., Wang, H. O. (2004). Fuzzy control systems design and analysis: a linear matrix inequality approach. John Wiley Sons.

[16] Sharp, Robin S, "The stability and control of motorcycles", Journal of mechanical engineering science, vol. 13,pp 316-329, 1971.

[17] V. Cossalter, Motorcycle Dynamics. Lulu. com, 2006.

[18] Darouach, M., Zasadzinski, M., Xu, S. J. (1994). Full-order observers for linear systems with unknown inputs. IEEE transactions on automatic control, 39(3), 606-609.

[19] Patton, R. J., Chen, J., Lopez-Toribio, C. J. (1998). Fuzzy observers for nonlinear dynamic systems fault diagnosis. In Decision and Control, 1998. Proceedings of the 37th IEEE Conference on (Vol. 1, pp. 84-89). IEEE.

[20] Ichalal, D., Marx, B., Ragot, J., Maquin, D. (2015). Unknown input observer for LPV systems with parameter varying output equation. IFAC-PapersOnLine, 48(21), 1030-1035.

[21] Damon, P. M., Dabladji, H., Ichalal, D., Nehaoua, L., Arioui, H., Mammar, S. (2016, September). Lateral motorcycle dynamics and rider action estimation: An lpv unknown input observer approach. In Control Applications (CCA), 2016 IEEE Conference on (pp. 711-716). IEEE.

[22] Damon, P. M., Ichalal, D., Nehaoua, L., Arioui, H. (2017). Lateral Steering Dynamics Estimation for Single Track Vehicle: Experimental Tests. IFAC-PapersOnLine, 50(1), 3400-3405. 